American Journal of Applied Sciences 9 (1): 71-74, 2012

ISSN 1546-9239

(C) 2012 Science Publications

\title{
Quality of Life of the Elderly Residents
}

\author{
${ }^{1}$ Fereshteh Farzianpour, ${ }^{2}$ Shayan Hosseini, ${ }^{3}$ Maryam Rostami, \\ ${ }^{4}$ Shahram B. Pordanjani and ${ }^{5}$ Sayed M. Hosseini \\ ${ }^{1}$ Department Health Management and Economics, \\ School of Public Health, Tehran University of Medical Sciences, Tehran, Iran \\ ${ }^{2}$ Department Management of power and Energy, \\ Amirkabir University, Tehran, Iran \\ ${ }^{3}$ Department Nursing and Midwifery, \\ Azad University of Masjed Solaiman, Iran, \\ ${ }^{4}$ Department Nursing and Midwifery, \\ School of Nursing and Midwifery, Ahwaz Jundishapur University, Iran \\ ${ }^{5}$ Department Epidemiology and Statistic, \\ School of Public Health, Tehran University of Medical Sciences, Tehran, Iran
}

\begin{abstract}
Problem statement: A preliminary investigation of the Orem self-care training effects on the Quality Of Life (QOL) of the health care centers' elderly residents in the city of Masjed Solaiman, Iran. Approach: The Orem self-care training was administered to 349 elderly free of chronic disorders (aged 65 and older). The eligible participants were selected from 11 health centers. The training was administered by research assistants. The cumulative training time was four months. The training consisted of six 40 min sessions. The Short Form Health Survey (SF-36) was used to compare QOL scores before and after the training using a paired t-test. Results: The overall health survey scores improved significantly after the training ( $\mathrm{p}<0.001$, mean increase from 49.2-59.0). The physical role score increased from 48.7-57.4; physical functioning from 55.3-66.3; mental health from 52.1-62.2; social functioning from 57.4-68.5; body pain tolerance from 47.4-53.3; vitality from 47.0-57.6 and emotional role from 47.2-61.9. Conclusion: The Orem self-care training can improve the QOL scores in the elderly population. Additional studies including a random selected subjects and paired controls to are required to validate our preliminary findings. A significant statistical bias could have been introduced by the lack of controls and accounting for other variables such as sex and literacy.
\end{abstract}

Key words: Orem self-care model, quality of life, elderly, chronic disorders, isolation, social and economic challenges

\section{INTRODUCTION}

Aging individuals experience reduced physical stamina and mental sharpness. Elderly experience increased incidence of emotional disturbances resulting from loneliness, impaired sexual activity, chronic metabolic disorders and cancer. The number of Iranian elderly continues to grow (Farzianpour et al., 2011). In 2006 United Nations (UN) reported that Iran is the home for 4,562,000 citizen aged 60 or older $(6 \%$ of the total population). It is estimated that by 2050 this number will rise to $26,393,000$, i.e., $26 \%$ of the total population (Bornardz, 1997; Kun, 2001). The elderly Quality Of Life (QOL) is also challenged by poor economic, cultural, educational and health care conditions. Inadequate social interactions and health literacy also contribute to QOL decline (Bornardz, 1997; Kun, 2001; Mellor et al., 2008; Gureje et al., 2006; Donmez et al., 2005; Lawes, 2002; Bruce, 1999; Rao, 2000). For example patients with limited physical activity following strokes suffer from depression (Rao, 2000). There is a negative correlation between the elderly inability to perform daily activities and their cognitive functioning. Patients with limited ambulatory capacity, due to chronic respiratory problems, also exibit poor Quality Of Life (QOL) scores (Chan et al., 1998; Paluska and Schwenk, 2000; Fassino et al., 2002). One-fifth of the elderly population is unable to perform daily routine activities and require physical support (Hellstrom et al., 2004).

Corresponding Author: Fereshteh Farzianpour, Department Health Management and Economics, School of Public Health, Tehran University of Medical Sciences, Iran 
Several authors reported premature physical aging among some of the Iranian elderly (Aghamolaei et al., 2010; Bornardz, 1997; Kun, 2001; Mellor et al., 2008; Gureje et al., 2006). Preventive programs targeting the QOL of the Iranian elderly should be considered. A significant improvement in the overall elderly QOL following the Orem model and self-care training was reported (Klainin and Ounnapiruk, 2010; Ware et al., 1993). This study was undertaken to test the benefit of such training on the QOL of the elderly in Masjed Sulaiman.

\section{MATERIALS AND METHODS}

A preliminary intervention study on a "healthy" sample of elderly residents from 11 health centers in Masjed Solaiman, Iran was conducted. The study was approved by the Ethics Committee of Tehran University Medical Sciences (TUMS) IRB No. 8819719/7/2007 and by the Ethics Committee of the Nursing College of Joundi Shapur Medical Science University, Ahvaz, Iran. The researchers described the purpose of the study and potential risks/benefits to the health centers' managers and all the prospective participants. All subjects were required to sign a consent form.

From 6,188 health center residents, 349 were found eligible for inclusion into the study (169 males and 180 females). The criteria for selecting participants were (a) a minimum age of 65, (b) no debilitating chronic disorders including mental disease, (c) living in Masjed Solaiman and (d) a consent to participate. Exclusion criteria were the presence of heart, lung, metabolic and musculoskeletal system diseases. No controls were included in the study.

The participants received a 4-month Orem selftraining program. Behavior modifications to improve the QOL such as a balanced diet, low salt intake, weight adjustments, adherence to prescribed medications, physical activities (regular walking, climbing stairs and using exercise devices), smoking cessation and daily hygiene and self-care were included in the training. The training consisted of lectures and practical demonstration sessions. All sessions were handled by the research assistants. A total of six $40 \mathrm{~min}$ sessions thought to all participants over a four months period (2001-2008). The research assistants were trained under the authors' supervision a month before the beginning of the study. A standard training protocol, described previously, was used (Tajvar and Farzianpour, 2004).

The QOL was measured using the Medical Outcomes Study 36-Item Short Health Survey Form (SF36) (Ware et al., 1994; Beddhu et al., 2000; Valderrabano et al., 2001). This instrument has been widely used in both Iran and other countries (Taghipoiur et al., 2009). The validity of the Persian language translation of the SF-36 questionnaire has been reported in previous studies (Montazeri et al., 2005).

The Short health Survey (SF-36) contains 36 items and is divided into eight subscales that assess General Health (GH): Physical Function (PF), health problems resulting in limitations of physical activities; Physical Role (PR), physical disability resulting in limitations of usual role activities; Body Pain (BP), daily activities influenced by pain; General Health (GH), selfperception of overall health; Vitality (VT), overall energy level or lack thereof; Emotional Role (ER), emotional problems resulting in limitations of usual role activities, Mental Health (MH), psychological well-being and stress and Social Function (SF). The eight dimensions of the health survey were included either into mental or physical categories. Each subscale score was assigned a value between 0 and 100. QOL scores higher than 50 indicated a better and scores lower than 50 indicated a worse general health status.

Statistical analyses: The Cronbach alpha coefficient of reliability test score for the SF-36 survey was estimated at 0.85. Descriptive indices such as frequency, percentage, mean and Standard Deviations (SD) were used to present data. A paired $t$-test was used to analyze the data before and after the educational program. All analyses were carried out using SPSS software for Windows (Ver. 13.0).

The required number of participants for our study was computed using $\mathrm{n}=\frac{\left(\mathrm{Z}_{1-\alpha}+\mathrm{Z}_{1-\beta}\right)^{2} \times \sigma \frac{2}{\mathrm{~d}}}{\mathrm{~d}}$ (Kirkwood and Sterne, 2001) to detect a mean change of at least 5 units on the SF-36 score after educational intervention $(\bar{d}=5)$. For $\alpha=\beta=0.05$ and $\sigma \frac{2}{d}=625$, the sample size was computed as 325 . However, 349 (about $6 \%$ ) elderly (aged $\varepsilon 65$ ) who met the eligibility criteria were selected from all 11 health centers.

\section{RESULTS}

The demographic characteristics and smoking habits of the participants are presented in Table 1. The mean age of the participants was 74.51 (SD +/6.81, range, 65-92). Total $169(48.6 \%)$ of the participants were illiterate and most $(241,69.1 \%)$ were married and non-smokers $(282,80.8 \%)$.

Comparisons of the overall mean and its eight subscales of SF-36 are presented in Table 2. Significant improvements in all dimensions of the post-training QOL were measured $(\mathrm{p}<0.001)$. 
Am. J. Applied Sci., 9 (1): 71-74, 2012

Table 1: Selected demographic indicators of the participants

\begin{tabular}{|c|c|c|}
\hline Indicators Age & Mean $=74.5(\mathrm{~N})$ & $\mathrm{SD}=6.8(\%)$ \\
\hline \multicolumn{3}{|l|}{ Gender } \\
\hline Male & 169 & 48.4 \\
\hline Female & 180 & 51.3 \\
\hline \multicolumn{3}{|l|}{ Marital status } \\
\hline Married & 241 & 69.1 \\
\hline Single & 8 & 1.7 \\
\hline Separated & 10 & 2.3 \\
\hline Widow & 90 & 25.5 \\
\hline \multicolumn{3}{|l|}{ Education } \\
\hline Illiterate & 169 & 48.6 \\
\hline Primary school & 102 & 29.3 \\
\hline Secondary school & 54 & 15.5 \\
\hline Diploma & 13 & 3.7 \\
\hline Higher than diploma & 10 & 2.5 \\
\hline \multicolumn{3}{|l|}{ Residency status } \\
\hline Sanatorium & 1 & 0.3 \\
\hline Home & 347 & 99.7 \\
\hline \multicolumn{3}{|l|}{ Occupation } \\
\hline Staff & 47 & 13.5 \\
\hline Worker & 40 & 11.5 \\
\hline Farmer & 25 & 7.2 \\
\hline Military & 15 & 4.3 \\
\hline At home & 140 & 40.1 \\
\hline Other & 82 & 23.5 \\
\hline \multicolumn{3}{|l|}{ Income source } \\
\hline Retirement salary & 101 & 29.2 \\
\hline Insurance & 64 & 18.5 \\
\hline Family & 72 & 20.8 \\
\hline No income & 90 & 25.7 \\
\hline Governmental charity & 20 & 5.6 \\
\hline \multicolumn{3}{|l|}{ Smoking habits } \\
\hline Non-smoker & 282 & 80.8 \\
\hline$<10$ & 7 & 2.1 \\
\hline $10-20$ & 25 & 7.3 \\
\hline$>20$ & 34 & 9.7 \\
\hline
\end{tabular}

Table 2: Mean and SD of the Health Survey categories before and after the training

\begin{tabular}{llll}
\hline Subscales & $\begin{array}{l}\text { Before } \\
\text { Mean (SD) }\end{array}$ & $\begin{array}{l}\text { After } \\
\text { Mean (SD) }\end{array}$ & p-value \\
\hline General health & $36.7(17.8)$ & $43.1(17.9)$ & $<0.001$ \\
Physical role & $48.7(26.7)$ & $57.4(31.4)$ & $<0.001$ \\
Physical functioning & $55.3(18.0)$ & $66.3(40.4)$ & $<0.001$ \\
Mental health & $52.1(17.5)$ & $62.2(16.7)$ & $<0.001$ \\
Social functioning & $57.4(19.0)$ & $68.5(54.1)$ & $<0.001$ \\
Body pain & $47.4(21.8)$ & $53.3(20.0)$ & $<0.001$ \\
Vitality & $47.0(12.7)$ & $57.6(11.6)$ & $<0.001$ \\
Emotional role & $47.2(18.3)$ & $61.9(40.6)$ & $<0.001$ \\
Overall mean & $49.2(16.0)$ & $59.0(17.6)$ & $<0.001$ \\
\hline
\end{tabular}

\section{DISCUSSION}

Our findings support the reported results of the positive counseling model outcomes on the QOL of the elderly in the cities of Zahedan and Shahinshar, Iran.

The mean QOL scores in other countries are much higher than the results obtained in Iran (Tasi et al., 2004). QOL is influenced by many variables, including age, culture, physical, environmental and socioeconomic status which is difficult to control. Patient and family education and cooperation are also major factors affecting the QOL. Therefore, raising elderly awareness and knowledge regarding positive behavioral modifications that affect QOL is important.

\section{CONCLUSION}

Elderly who have an active lifestyle exhibit better QOL, are less depressed, more satisfied and socially interactive. The elderly who are capable of performing their daily activities are also less likely to become depressed (Secondo et al., 2002).

Programs addressing financial support systems, social security, treatment insurance, etc. should be considered important factors in promoting successful aging and QOL for the Iranian elderly. Outreach and educational training programs for family members caring for the elderly can benefit the overall wellbeing of this growing population (Darani et al., 2010).

Additional studies using a larger segment of Iranian aging population and cross comparison among similar studies conducted in different regions of Iran, are required to develop the supporting evidence which could benefit the aging population.

\section{REFERENCES}

Aghamolaei, T., S.S. Tavafian and S. Zare, 2010. Health related quality of life in elderly people living in Bandar Abbas, Iran: A population-based study. Acta Med. Iran, 48: 185-191. PMID: 21137656

Beddhu, S., F.J. Bruns, M. Saul, P. Seddon and M.L. Zeidel, 2000. A simple comorbidity scale predicts clinical outcomes and costs in dialysis patients. Am. J. Med., 108: 609-613. DOI: 10.1016/S00029343(00)00371-5

Bornardz, J., 1997. Family Studies: An Introduction. 1st Edn., Routledge, Tehran, ISBN: 0415164699, pp: 229.

Bruce, M.L., 1999. The Association between Depression and Disability. Am. J. Geriatr. Psychiatry, 7: 8-11. DOI: 10.1097/00019442199902000-00002

Chan, K.M., W.S. Pang, C.H. Ee, Y.Y. Ding and P. Choo, 1998. Self-perception of health among elderly community dwellers in Singapore. Annals Acad. Med. Singapore, 27: 7-461. PMID: 9588267

Darani, F.M., H.M. Riji, H. Abedi, S. Ahmad and L. Latif, 2010. How Iranian families response to the conditions affecting elderly primary health care. Res. J. Biol Sci., 5: 420-429. DOI: 10.3923/rjbsci.2010.420.429 
Donmez, L., Z. Gokkoca and N. Dedeoglu, 2005. Disability and its effects on quality of life among older people living in antalya city center, Turkey. Arch. Gerontol. Geriatr., 40: 213-223. DOI: 10.1016/j.archger.2004.08.006

Farzianpour, F., B. Pirozi, M. Arab, S.M. Hosseini and B.A. Fayaz, 2011. Quality of Life of the Elderly Residents in Marivan. MSc Thesis, Department Health Management and Economics, 5School of Public Health, Tehran University of Medical Sciences, Tehran, Iran.

Fassino, S., P. Leombruni, G.A. Daga, A. Brustolin and G.G. Rovera et al., 2002. Quality of life in dependent older adults living at home. Arch. Gerontol. Geriatr., 35: 9-20. DOI: 10.1016/S01674943(01)00210-2

Gureje, O., A. Ogunniyi, L. Kola and E. Afolabi, 2006. Functional disability in elderly Nigerians: Results from the Ibadan study of aging. J. Am. Geriatr. Soc., 54: 1784-1789. PMID: 17087709

Hellstrom, Y., G. Persson and I.R. Hallberg, 2004. Quality of life and symptoms among older people living at home. J. Adv. Nurs., 48: 584-593. PMID: 15548249

Kirkwood, B. and J. Sterne, 2001. Essentials of Medical Statistics. 2nd Edn., Wiley-Blackwell Ltd, Oxford, ISBN-10: 0865428719, pp: 288.

Klainin, P. and L. Ounnapiruk, 2010. A meta-analysis of self-care behavior research on elders in Thailand: An Update. Nurs. Sci. Q., 23: 156-163. DOI: $10.1177 / 0894318410362788$

Kun, L.G., 2001. Telehealth and the Global Health Network in the 21st Century. From homecare to public health informatics. Comput. Methods Programs Biomed., 64: 155-167. DOI: 10.1016/S0169-2607(00)00135-8

Lawes, D., 2002. A retrospective review of emergency admission for head injury in the over $75 \mathrm{~s}$. Injury, 33: 349-351. DOI: 10.1016/S0020-1383(02)00075$\mathrm{X}$

Mellor, D., S. Russo, M.P. McCabe, T.E. Davison and K. George, 2008. Depression training program for caregivers of elderly care recipients: Implementation and qualitative evaluation. $\mathrm{J}$. Gerontological Nurs., 34: 8-17. DOI: 10.3928/00989134-20080901-09
Montazeri, A., A. Goshtasebi, M. Vahdaninia and B. Gandek, 2005. The Short Form Health Survey (SF36): Translation and validation study of the Iranian Version. Quality Life Res., 14: 875-882. DOI: 10.1007/s11136-004-1014-5

Paluska, S.A. and T.L. Schwenk, 2000. Physical activity and mental health: Current concepts. Sports Med., 29: 167-180. DOI: 10.2165/00007256-200029030-00003

Rao, R., 2000. Cerebrovascular disease and late life depression: An age old association revisited. Int. J. Geriatr. Psychiatry, 15: 419-433. PMID: 10822241

Secondo, F., L. Paolo, A.D. Giovanni, B. Annalisa and G.R. Giovanni et al., 2002. Quality of life in dependent older adults living at home. Arch. Gerontol. Geriatr., 35: 9-20. DOI: 10.1016/S01674943(01)00210-2

Taghipoiur, H., Y. Moharamzad, A.R. Mafi, A. Amini and M.M. Naghizadeh et al., 2009. Quality of life among veterans with war-related unilateral lower extremity amputation: A long-term survey in a prosthesis center in Iran. J. Orthop. Trauma, 23: 525-530. PMID: 19633463

Tajvar, M. and F. Farzianpour, 2004. Elderly health and a review on different aspects of their life. Tehran: Nasle Farda and Arjmand Press. ISBN 6947957440 Tehran-Iran

Tasi, S.Y., L.Y. Chi, L.S. Lee and P. Choa, 2004. Health-related quality of life among urban, rural, and island community elderly in Taiwan. J. Formosan Med. Assoc., 103: 196-204. PMID: 15124047

Valderrabano, F., R. Jofre and J.M. Lopez-Gomez, 2001. Quality of life in end-stage renal disease patients. Am. J. Kidney Dis., 38: 443-464. PMID: 11532675

Ware, J.E., K.K. Snow, M. Kosinski and B. Gandek, 1993. SF-36 Health Survey: Manual and Interpretation Guide. 1st Edn., The Health Institute, New England Medical Center, Boston, MA.

Ware, J.E., M. Kosinski and S.D. Keller, 1994. SF-36 Physical and Mental Health Summary Scales: A User's Manual. 5th Edn., The Health Institute, New England Medical Center, Boston, MA., ISBN: 9781891810008, pp: 188. 